\title{
A case of membranous glomerulopathy associated with lung cancer and review of the literature
}

\author{
AYDIN AYTEKIN $^{1}$, AHMET OZET ${ }^{1}$, IREM BILGETEKIN ${ }^{1}$, \\ BETUL OGUT $^{2}$, AYDIN CILTAS ${ }^{1}$ and MUSTAFA BENEKLI ${ }^{1}$ \\ ${ }^{1}$ Department of Internal Medicine, Division of Medical Oncology; \\ ${ }^{2}$ Department of Pathology, Gazi University Faculty of Medicine, Ankara 06560, Turkey
}

Received August 2, 2016; Accepted March 15, 2017

DOI: $10.3892 /$ mco.2017.1295

\begin{abstract}
Membraneous nephropathy (MN) is the most commonly occurring nephrotic syndrome in adults as well as the most common paraneoplastic nephropathy associated with solid tumors, and it is mostly associated with gastrointestinal system and lung carcinomas. Accurate diagnosis is important as the treatment of paraneoplastic glomerulonephritis is very varied from that of idiopathic ones. In the current report, a case of a patient that was referred with proteinuria and edema and was diagnosed with lung cancer, and responded markedly to treatment of malignancy, with improvement of MN, is presented. Active cancer is present in all patients with paraneoplastic MN. In numerous patients, the paraneoplastic $\mathrm{MN}$ and cancer diagnoses are made within one year of each other. The treatment of paraneoplastic syndromes is usually associated with the treatment of primary malignancy. There are conflicting data on which treatment modality is more suitable. In conclusion, further studies are required in order to determine the actual incidence of cancer in patients with nephropathy, explain the physiopathological association between cancer and nephropathy and to determine the most suitable treatment approaches.
\end{abstract}

\section{Introduction}

Nephrotic syndrome is one of the most easily diagnosed kidney diseases due to its established criteria. It is a clinical entity characterized by proteinuria $>3.5 \mathrm{~g} / 1.73 \mathrm{~m}^{2}$ for $24 \mathrm{~h}$ along with hypoalbuminemia, edema and hyperlipidemia. In glomerular diseases presenting with nephrotic syndrome, there is a defect in glomerular filtration barrier (1). Membranous nephropathy $(\mathrm{MN})$, is a pathological entity characterized by

Correspondence to: Dr Aydin Aytekin, Department of Internal Medicine, Division of Medical Oncology, Gazi University Faculty of Medicine, Ankara 06560, Turkey

E-mail: aydinaytekin@gazi.edu.tr

Key words: membranous glomerulopathy, lung cancer, adenocarcinoma, nephrotic syndrome, solid tumor diffuse thickening in the glomerular basement membrane, as viewed under light microscopy. Although $75 \%$ of MN cases are idiopathic, the remaining are associated with infections, malignancies, autoimmune diseases and drug toxicity (2).

The diagnosis of paraneoplastic nephropathy is made according to certain criteria: Clinical and histological remission with complete removal of the tumor or complete remission with chemotherapy, nephrological relapse concurrent with the recurrence of malignancy and physiological connection between two diseases including tumor antigens and antitumor antibodies along with immune deposits (3). MN is the nephrotic syndrome occurring most frequently in adults and the paraneoplastic nephropathy detected most commonly in association with solid tumors (4), and it is most commonly associated with the gastrointestinal system and lung adenocarcinomas (1). Accurate diagnosis is important as the treatment of paraneoplastic glomerulonephritis is different from that of idiopathic nephropathies. The aim of the present report was to present the case of a patient who was referred with proteinuria and edema, was diagnosed with MN and subsequently lung cancer and responded markedly to treatment of malignancy with improvement of MN.

\section{Case report}

A 62 year old male patient with a history of coronary artery disease and a history of smoking (36 pack-years) underwent kidney biopsy in July 2012 due to widespread edema and $11.8 \mathrm{~g} /$ day proteinuria, high creatinine levels $(1.63 \mathrm{mg} / \mathrm{dl}$; normal range 0.8-1.3), hyperlipidemia (total cholesterol $478 \mathrm{mg} / \mathrm{dl}$. normal range 110-200; low density lipoprotein, $359 \mathrm{mg} / \mathrm{dl}$, normal range 60-130) and hypoalbuminemia (albumin $1.6 \mathrm{~g} / \mathrm{dl}$, normal range 3.5-5). Histological analysis of the kidney biopsy was consistent with membranous nephropathy (Fig. 1 reveals thickening in the glomerular basement membrane detected with hematoxylin and eosin staining and Fig. 2 highlights subepithelial deposits detected using immunohistochemistry staining for immunoglobulin G). In etiological investigations, spiculated contoured lesions were detected in the lungs: a $17 \mathrm{~mm}$ lesion in the right lung apex and a $22 \mathrm{~mm}$ lesion in the upper lobe posterior segment. Subsequently, the patient underwent positron emission tomography-computed tomography (PET-CT) investigation and increased 18-fludeoxyglucose 

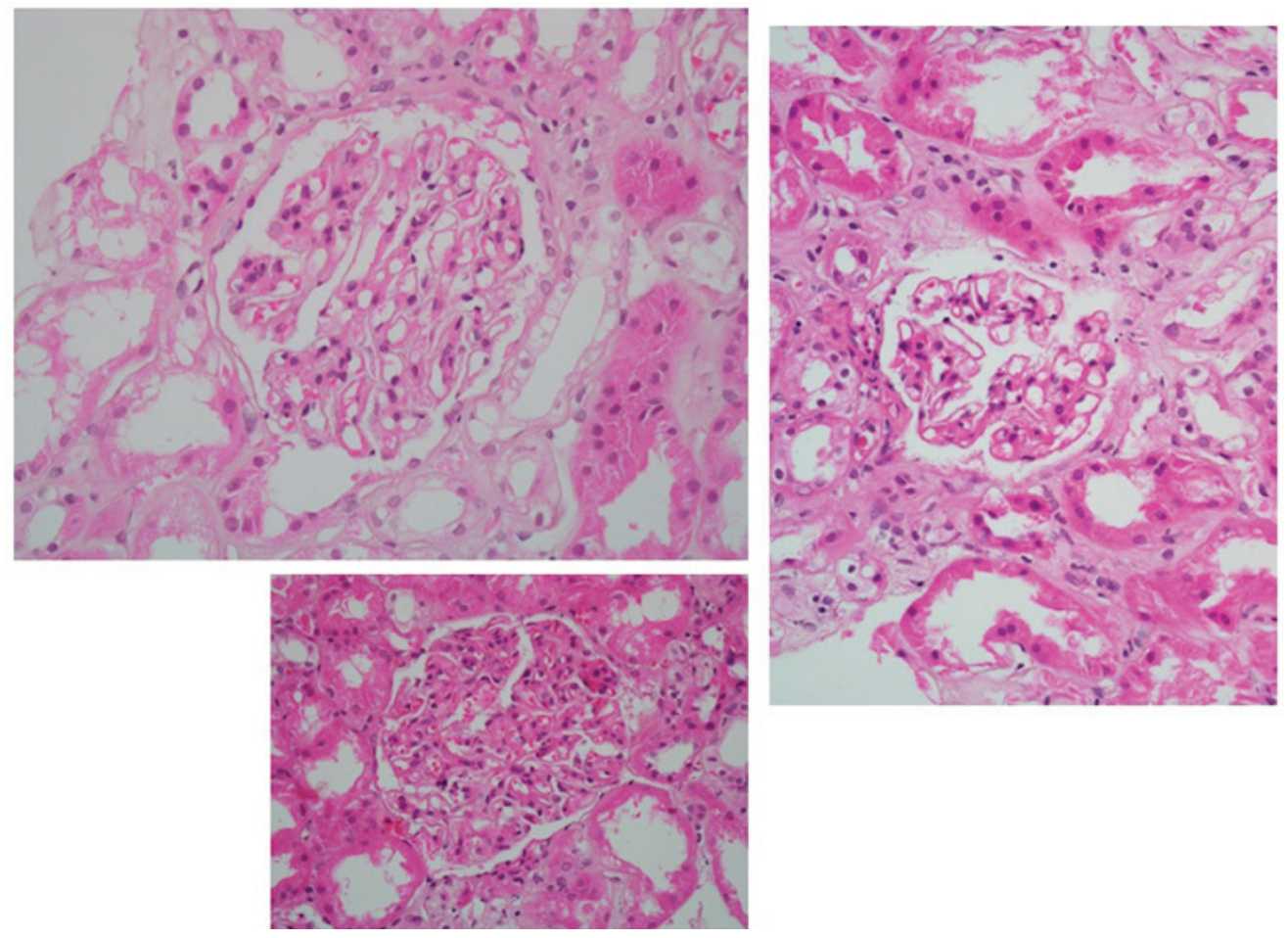

Figure 1. Thickening in glomerular basement membrane. Hematoxylin and eosin staining; magnification, x400.

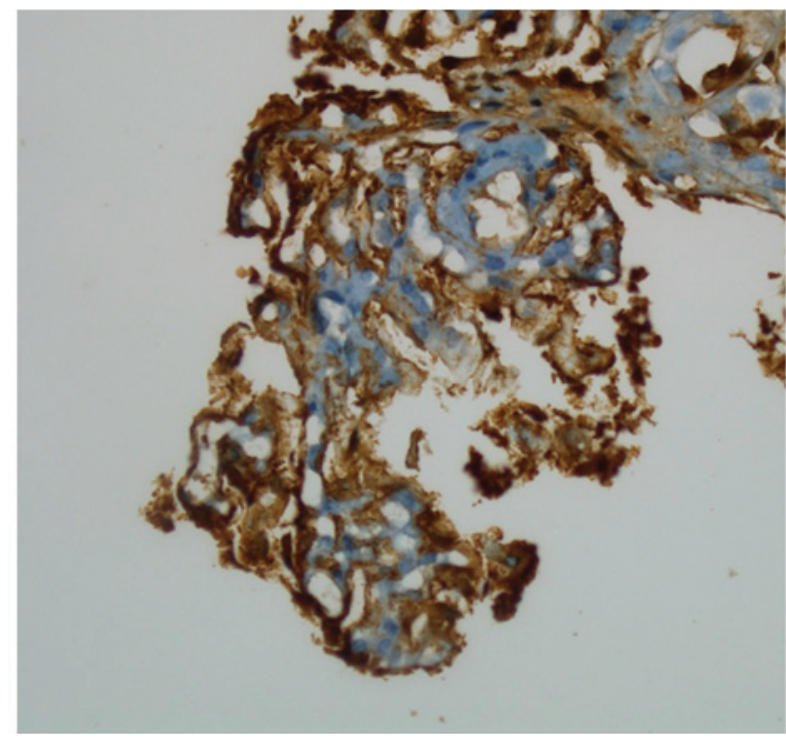

Figure 2. Granular staining as determined by immunohistochemistry in subepithelial deposits in the glomerular basement membrane. Immunoglobulin G; magnification x400.

(18-FDG) involvement was identified in the mediastinal lymphadenopathies (LAPs), nodules in the apex and posterior segment of upper lobe of right lung. Pathological sampling was performed using fiber optic bronchoscopy, which initially detected adenocarcinoma. The patient underwent wedge resection and lymph node dissection and was diagnosed with T3N2M0 stage disease according to the TNM classification of malignant tumors (5), and stage $3 \mathrm{~B}$ lung adenocarcinoma according to the American Joint Committee on Cancer 2010 criteria (6). Adjuvant radiotherapy (with paclitaxel $45 \mathrm{mg} / \mathrm{m}^{2}$ on day 1 and carboplatin AUC 2 on day 1 , every 7 days during thoracic radiotherapy) and then paclitaxel $\left(175 \mathrm{mg} / \mathrm{m}^{2}\right.$ on day $1+$ carboplatin AUC-5 on day 1, every 21 days for 6 cycles) chemotherapy was administered. Two months following surgery, the proteinuria had regressed to $175 \mathrm{mg} /$ day. After six months (the end of chemotherapy), the proteinuria had increased to $817 \mathrm{mg} /$ day. PET-CT investigation was performed and the apical region of the upper lobe of the right lung and upper posterior segment of the right lung increased pathologic 18-FDG uptake were identified and, considering recurrence, a right lung upper lobectomy + mediastinal lymph node dissection were performed. Pathological examination detected residual carcinoma areas and 20 lymph nodes exhibiting reactive changes. The patient was followed without intervention and proteinuria decreased to $92 \mathrm{mg} / \mathrm{day}$. MN was observed to be in complete remission. After six months of follow-up, due to suspicion of recurrence, PET-CT was performed and increased 18F-FDG involvement was observed in the right lung middle lobe and the anterior and posterior of the right hemithorax in the diaphragmatic pleura and 9th costa. The patient is in complete remission for nephrotic syndrome and has no associated symptoms. Nivolumab treatment was commenced for recurrent metastatic lung carcinoma. Patient received 6 courses of treatment and has been in complete remission for MN for 33 months.

\section{Discussion}

Two important risk factors differentiating paraneoplastic membranous nephropathy from idiopathic $\mathrm{MN}$ are an age $>65$ and a history of smoking $>20$ pack-years. All patients with paraneoplastic $\mathrm{MN}$ also have active cancer. The age range of patients with paraneoplastic MN was 55-65 and the median 
age was 60 . In the majority of patients, the diagnosis of paraneoplastic $\mathrm{MN}$ and cancer are made within 1 year; however, in half of patients, cancer symptoms are present at the diagnosis of MN (7). Malignancy is detected in $11-13 \%$ of patients with nephrotic syndrome (1).

Lee et al (8) reported that of 101 nephrotic syndromes, 11 were associated with malignacy and of these 8 presented with MN. Eagan (9) reported that 28/67 patients with a nephrotic syndrome-associated malignancy had lung cancer.

In the recent study of Lefaucheur et al (10) on 240 patients with $\mathrm{MN}$, cancer prevalence was determined at the rate of $10 \%$ in patients diagnosed with $\mathrm{MN}$, and this study had one of the largest patient series published so far. It was stated that this association becomes more marked with age; comparisons were made with general population and it was established that cancer prevalence was 10-fold higher in patients with $\mathrm{MN}$ for both sexes and all age groups. It was also reported that carcinomas (20 cases, 83.3\%) account for the majority of $\mathrm{MN}$-associated tumors, and the most common locations were lung ( 8 patients, 4 squamous cell and 4 adenocarcinoma) and prostate (5 patients) (10). Another important point stressed in this previous study was that proteinuria decreased with tumor regression. Similarly, in other previous studies, the prevalence of cancer in patients with MN was reported to be $5-22 \%(11,12)$.

In a recent meta-analysis (13), it was stated that majority of malignancies in MN patients are solid tumors (86\%, 73 cases) and hematological malignancies were observed in $14 \%$ of cases (12 cases). Lung cancer is the most common accompanying malignancy (22 cases, $26 \%$ ) followed by prostate cancer (13 cases, $15 \%$ ), colorectal cancer ( 9 cases, $11 \%$ ), breast cancer (6 cases, 7\%), stomach and esophagus cancer (5 cases, 6\%), bladder cancer (4 cases, $5 \%$ ) and cervical and uterine cancers (3 cases, 4\%). In the present study, similar to the study of Lefaucheur et al (10), MN cancer prevalence was reported to be $10 \%$ and the mean age of the patients was $66.35 \pm 6.75$.

The treatment of paraneoplastic syndromes is usually associated with the treatment of primary malignancy. There are conflicting data on which treatment modality is more appropriate. In a previous study, it was reported that complete surgical resection of small lesion lung cancers prevents the progression of nephrotic syndromes and decreases the excretion of urinary protein (14). In the present case, chemotherapy was administered following surgical intervention and nephropathy resolved during this process. Although it is not possible to say definitively which treatment was effective, following a second recurrence, the patient underwent only surgical intervention and proteinuria returned to the normal physiological range, which underscores the contribution of surgery. In a case report (15) including two patients with stage 3 squamous cell lung cancer, paraneoplastic syndrome improved with radiotherapy and it was reported that these patients may be administered radiotherapy. In another previous report, it was stated that chemotherapy led to the regression of nephrotic syndrome (16). In an additional case report, (17) it was stated that in a patient with stage 3 non small cell lung cancer, chemotherapy led to dramatic improvement in cancer and $\mathrm{MN}$, but $\sim 14$ months later, $\mathrm{MN}$ also recurred along with the recurrence in cancer. Unlike the aforementioned case, in our case, cancer recurrence was identified twice, and at the second recurrence, an increase in proteinuria was observed ( $817 \mathrm{mg} /$ day), but this returned to normal levels following surgery and $\mathrm{MN}$ coursed in remission.

In conclusion, further studies are required in order to determine the real incidence of cancer in paraneoplastic nephropathy patients, to explain the association between cancer and nephropathy and to determine the most effective treatment approaches.

\section{References}

1. Wągrowska-Danilewicz M and Danilewicz M: Nephrotic syndrome and neoplasia: Our experience and review of the literature. Pol J Pathol 62: 12-18, 2011.

2. Ponticelli C and Glassock RJ: Glomerular diseases: Membranous nephropathy-a modern view. Clin J Am Soc Nephrol 9: 609-616, 2014.

3. Burstein DM, Korbet SM and Schwartz MM: Membranous glomerulonephritis and malignancy. Am J Kidney Dis 22: 5-10, 1993.

4. Ronco PM: Paraneoplastic glomerulopathies: New insights into an old entity. Kidney Int 56: 355-377, 1999.

5. Goldstraw P, Crowley J, Chansky K, Giroux DJ, Groome PA, Rami-Porta R, Postmus PE, Rusch V and Sobin L; International association for the study of lung cancer international staging committee; participating institutions: The IASLC lung cancer staging project: Proposals for the revision of the TNM stage groupings in the forthcoming (seventh) edition of the TNM Classification of malignant tumours. J Thorac Oncol 2: 706-714, 2007.

6. Edge SB and Compton CC: The American Joint Committee on Cancer: The 7th edition of the AJCC cancer staging manual and the future of TNM. Ann Surg Oncol 17: 1471-1477, 2010.

7. Lien YH and Lai LW: Pathogenesis, diagnosis and management of paraneoplastic glomerulonephritis. Nat Rev Nephrol 7: 85-95, 2011.

8. Lee JC, Yamauchi H and Hopper J: The association of cancer and the nephrotic syndrome. Ann Intern Med 64: 41-51, 1966.

9. Eagen JW: Glomerulopathies of neoplasia. Kidney Int 11: 297-303, 1977.

10. Lefaucheur C, Stengel B, Nochy D, Martel P, Hill GS, Jacquot C and Rossert J; GN-PROGRESS Study Group: Membranous nephropathy and cancer: Epidemiologic evidence and determinants of high-risk cancer association. Kidney Int 70: 1510-1517, 2006.

11. Cahen R, Francois B, Trolliet P, Gilly J and Parchoux B: Aetiology of membranous glomerulonephritis: A prospective study of 82 adult patients. Nephrol Dial Transplant 4: 172-180, 1989.

12. Zech P, Colon S, Pointet P, Deteix P, Labeeuw M and Leitienne P: The nephrotic syndrome in adults aged over 60: Etiology, evolution and treatment of 76 cases. Clin Nephrol 17: 232-236, 1982.

13. Leeaphorn N, Kue-A-Pai P, Thamcharoen N, Ungprasert P, Stokes MB and Knight EL: Prevalence of cancer in membranous nephropathy: A systematic review and meta-analysis of observational studies. Am J Nephrol 40: 29-35, 2014.

14. Coltharp WH, Lee SM, Miller RF and Averbuch MS: Nephrotic syndrome complicating adenocarcinoma of the lung with resolution after resection. Ann Thorac Surg 51: 308-309, 1991.

15. Shikata Y, Hayashi Y, Yamazaki H, Shikata K and Makino H: Effectiveness of radiation therapy in nephrotic syndrome associated with advanced lung cancer. Nephron 83: 160-164, 1999.

16. Boon ES, Vrij AA, Nieuwhof C, van Noord JA and Zeppenfeldt E: Small cell lung cancer with paraneoplastic nephrotic syndrome. Eur Respir J 7: 1192-1193, 1994.

17. Crawford AR, Dworkin L, Leonard K, Khurshid H and Hepel JT: Recurrence of paraneoplastic membranous glomerulonephritis following chemoradiation in a man with non-small-cell lung carcinoma. Rare Tumors 5: 62-64, 2013. 